\title{
Conformally covariant vector-spinor field in de Sitter space
}

\author{
N. Fatahi ${ }^{1, \mathrm{a}}$, M. V. Takook ${ }^{1, \mathrm{~b}}$, M. R. Tanhayi ${ }^{2, \mathrm{c}}$ \\ ${ }^{1}$ Department of Physics, Science and Research Branch, Islamic Azad University, Tehran, Iran \\ ${ }^{2}$ Department of Physics, Islamic Azad University, Central Tehran Branch, Tehran, Iran
}

Received: 30 May 2014 / Accepted: 27 September 2014 / Published online: 29 October 2014

(c) The Author(s) 2014. This article is published with open access at Springerlink.com

\begin{abstract}
In this paper, we study conformally invariant field equations for a vector-spinor ( $\operatorname{spin}-\frac{3}{2}$ ) field in the de Sitter space-time. The solutions are also obtained in terms of the de Sitter-Dirac plane waves. The related two-point functions are calculated in both the de Sitter ambient space formalism and intrinsic coordinates. In order to study the conformal invariance, Dirac's six-cone formalism is utilized in which the field equations are expressed in a manifestly conformal way in 4+2-dimensional conformal space and then followed by a projection to the de Sitter space.
\end{abstract}

\section{Introduction}

Recent astrophysical data indicate that our universe is undergoing an accelerated expansion [1] and in a first approximation the background space-time can be well described by the de Sitter (dS) space. The dS universe is a maximally symmetric curved space with the same degrees of symmetry as the Minkowskian space. It also plays an important role in the inflation scenario where the cosmic dynamics is assumed to be dominated by a term that acts like a cosmological constant [2]. Thus, constructing a formulation of dS quantum field theory seems to be important; this has been studied by many authors [3-8]. The high level of symmetry in this curved space-time can be a guideline for constructing the quantum fields and the underlining symmetry of the background may be well established by group theory. Actually revealing the group theoretical and geometrical structures underlying the theory is of great importance. We use this idea to study the higher-spin free field in the de Sitter space. Basically studying the free higher-spin fields may shed some light on our better understanding of the interactions in higher-spin field theories. As is well known, the construction of a consistent interacting

\footnotetext{
a e-mail: fatahi@iauksh.ac.ir

be-mail:mtakook@yahoo.com; takook@razi.ac.ir

c e-mail: m_tanhayi@iauctb.ac.ir
}

theory of higher-spin fields is one of the oldest long-standing problems in theoretical physics of particles and fields.

The higher-spin field equations have been considered in [9]. It was proved that a given field of rest mass $m$ and spin $s \geq 1$ can be represented by a completely symmetric multispinor [10]. Manifestly covariant massless fields for halfinteger spin in anti-de Sitter background have been studied in [11-13]. The massive spin- $\frac{3}{2}$ field in dS space has been considered in [14] and the extension to the massless fields is done in [15]. Here, within the language of the group theory in de Sitter space, we study the conformal invariance of a vector-spinor field by finding the field equations, solutions, and two-point function.

As is well known, the massless fields which live on the light cone, are conformally invariant [16], generally, all massless gauge fields in four-dimensional constant curvature spaces are conformally invariant at the level of degrees of freedom. This indicates that the respective representations of the (A)dS or the Poincare group can be extended to the representations of the conformal group [8]. The details for higher-spin gauge fields can be found in [17-19]. For our purpose, in dS space-time the conformal invariance may be understood by the concept of light-cone propagators. Noting that in $\mathrm{dS}$ space the mass is not a well-defined invariant parameter for a set of observers transforming under the $\mathrm{dS}$ group $S O(1,4)$, the masslessness is used in reference to the conformal invariance (propagation on the dS light cone). From the group theoretical point of view, the massive representations refer to those that coincide with the massive representation of the Poincare group in the zero curvature limit (Appendix A). Our motivation to study the spin-vector conformal invariance field equation in $\mathrm{dS}$ space is that in the supergravity model this field is the partner of the gravitational field and it must propagate on the dS light cone.

In flat space, according to group representation theory and Wigner's interpretation of the elementary systems, the field operators of any kind of spin transform according to the unitary irreducible representation (UIR) of the Poincaré group 
(the kinematical group of Minkowski space), and their corresponding field equations are well established by the Casimir operators. Being interested in the symmetry of space, the isometry group of the dS space is $S O_{0}(1,4)$ which may be viewed as a deformation of the proper orthochronous Poincaré group. There are two Casimir operators in the dS group and it has been shown that the massive scalar, vector, and spin-2 fields can be associated with the UIRs of the dS group [5,20-23]. The massless fields can be associated with an indecomposable representation of the dS group [24]. The covariant quantum field theory for massive and massless conformally coupled scalar field in dS space have been studied in $[5,25]$ and also for the massless minimally coupled scalar field in [26]. In Refs. [27,28], one can find the conformally covariant quantization of the gauge field in $\mathrm{dS}$ space.

The main goal of this paper is to study conformal invariance of the spin-3/2 field in the de Sitter space, we use the group theoretical approach and hence the de Sitter invariance becomes manifest. The paper is organized in seven sections: The conformally invariant spin- $\frac{3}{2}$ field equations are obtained in Sects. 2 and 3. The solutions are found in Sect. 4, as will be shown, they can be written in terms of the de Sitter-Dirac plane waves. In Sects. 5 and 6, we find the two-point function in both embedding space namely $4+1$ ambient space of $\mathrm{dS}$ and also in four-dimensional intrinsic space. We discuss the results in the conclusion part. Finally, some useful relations are presented in the appendices.

\section{Massless spinor field equations in de Sitter space}

The dS metric is the unique solution of Einstein's equation in vacuum with positive cosmological constant $\Lambda=3 H^{2}$, in which dS space may be visualized as the hyperboloid embedded in a five-dimensional Minkowski space,

$X_{H}=\left\{x \in \mathbb{R}^{5} ; x^{2}=\eta_{\alpha \beta} x^{\alpha} x^{\beta}=-H^{-2}\right\}$,

$H$ is the Hubble constant, hereafter, for the sake of simplicity, we set $H=1$. The de Sitter metric is

$\mathrm{d} s^{2}=\left.\eta_{\alpha \beta} \mathrm{d} x^{\alpha} \mathrm{d} x^{\beta}\right|_{x^{2}=-1}=g_{\mu \nu}^{\mathrm{d} S} \mathrm{~d} X^{\mu} \mathrm{d} X^{\nu}$

where $\eta_{\alpha \beta}=\operatorname{diag}(1,-1,-1,-1,-1), \alpha, \beta=0,1,2,3,4$, and $\mu, v=0,1,2,3$. We use $x^{\alpha}$ for ambient space formalism whereas $X$ stand for de Sitter intrinsic coordinates. For simplicity the dot product is shown as $x^{\alpha} \partial_{\alpha} \equiv x \cdot \partial$. We define the transverse derivative in de Sitter space as $\partial_{\alpha}^{T} \equiv \theta_{\alpha \beta} \partial^{\beta}=\partial_{\alpha}+H^{2} x_{\alpha} x \cdot \partial$, where $\theta_{\alpha \beta}=\eta_{\alpha \beta}+H^{2} x_{\alpha} x_{\beta}$ is the projection operator and note that $x \cdot \partial^{T}=0$. Working in embedding space has two advantages; first it is close to the group theoretical language and second the equations are obtained in an easer way than they might be found in de Sitter intrinsic space.
There are two Casimir operators for dS group, these operators commute with all the action of the group generators and thus they are constant on each representation. In this section we briefly recall the notations of the Casimir operator and more details can be found in [20,29-33]. The second- and fourth-order Casimir operators are

$Q^{(1)}=-\frac{1}{2} L^{\alpha \beta} L_{\alpha \beta}, \quad Q^{(2)}=-W_{\alpha} W^{\alpha}$,

where $W_{\alpha}=\frac{1}{8} \epsilon_{\alpha \beta \gamma \delta \eta} L^{\beta \gamma} L^{\delta \eta}$ and $\epsilon_{\alpha \beta \gamma \delta \eta}$ is the antisymmetric tensor in the ambient space notation with $\epsilon_{01234}=1$. The generator of the de Sitter group is defined by

$L_{\alpha \beta}=M_{\alpha \beta}+S_{\alpha \beta}$,

where the "orbital" part is

$M_{\alpha \beta}=-i\left(x_{\alpha} \partial_{\beta}-x_{\beta} \partial_{\alpha}\right)=-i\left(x_{\alpha} \partial_{\beta}^{T}-x_{\beta} \partial_{\alpha}^{T}\right)$,

and the "spinoral" part $S_{\alpha \beta}$ which acts on the spinor field $(s=1 / 2)$ is [29]

$S_{\alpha \beta}=-\frac{i}{4}\left[\gamma_{\alpha}, \gamma_{\beta}\right]$.

In this case the five $4 \times 4$ matrices of $\gamma^{\alpha}$ are the generators of the Clifford algebra, which are constructed as

$\gamma^{\alpha} \gamma^{\beta}+\gamma^{\beta} \gamma^{\alpha}=2 \eta^{\alpha \beta}, \quad \gamma^{\alpha \dagger}=\gamma^{0} \gamma^{\alpha} \gamma^{0}$.

Based on spectrum of the possible values of the Casimir eigenvalues, the UIRs of the de Sitter group can be classified as the principal, complementary, and discrete series (Appendix A). In the principal series which belongs to the massive representation of the $\mathrm{dS}$ space and tends to the massive representation of the Poincaré group at the zero curvature limit, the eigenvalues of the Casimir operators can be written as $[30,31]$

$$
\begin{aligned}
& \left\langle Q_{s}^{(1)}\right\rangle=\left(\frac{9}{4}+v^{2}-s(s+1)\right), \\
& \left\langle Q_{s}^{(2)}\right\rangle=\left(\left(\frac{1}{4}+v^{2}\right) s(s+1)\right),
\end{aligned}
$$

where $s$ stands for the spin, $v$ is a real positive parameter. The second-order field equations can be written as ${ }^{1}$

$\left(Q_{s}^{(1)}-\left\langle Q_{s}^{(1)}\right\rangle\right) \psi=0$.

\footnotetext{
${ }^{1}$ Note that in writing the field equations we use only the second-order Casimir operator, because the fourth-order one leads to higher derivative equations,

$$
\begin{aligned}
Q^{(2)}= & -W_{\alpha} W^{\alpha}=\frac{1}{64} \epsilon_{\alpha \beta \gamma \delta \eta} \epsilon^{\alpha \beta \gamma \delta \eta}\left[M^{\beta \gamma} M^{\delta \eta} M_{\beta \gamma} M_{\delta \eta}\right. \\
& +M^{\beta \gamma} M^{\delta \eta} M_{\beta \gamma} S_{\delta \eta}+M^{\beta \gamma} M^{\delta \eta} S_{\beta \gamma} M_{\delta \eta} \\
& \left.+M^{\beta \gamma} M^{\delta \eta} S_{\beta \gamma} S_{\delta \eta}+\cdots\right] .
\end{aligned}
$$
}


For example for $s=1 / 2$, one has

$$
\left(Q_{\frac{1}{2}}^{(1)}-\frac{3}{2}\right) \psi=v^{2} \psi
$$

$\psi$ stands for a spinor field with arbitrary degree of homogeneity: $x \cdot \partial \psi=\sigma \psi$. The second-order Casimir operator for spin- $\frac{1}{2}$ is given by

$Q_{\frac{1}{2}}^{(1)}=-\frac{1}{2} M_{\alpha \beta} M^{\alpha \beta}-\frac{1}{2} S_{\alpha \beta} S^{\alpha \beta}-S_{\alpha \beta} M^{\alpha \beta}$,

where one can show

$\frac{1}{2} S_{\alpha \beta} S^{\alpha \beta}=\frac{5}{2}, \quad S_{\alpha \beta} M^{\alpha \beta}=-\frac{i}{2} \gamma_{\alpha} \gamma_{\beta} M^{\alpha \beta}=-\not x \not \partial^{T}$,

note that $\gamma_{\alpha} x^{\alpha} \equiv \not x$. After making use of the above relations, (2.8) can be written in terms of the scalar Casimir operator as follows:

$\left(Q_{0}+\not x \not \partial^{T}-4\right) \psi=v^{2} \psi$,

where $Q_{0}^{(1)}\left(\equiv Q_{0}\right)=-\frac{1}{2} M_{\alpha \beta} M^{\alpha \beta}$ is the spin-less Casimir operator. If one defines the de Sitter-Dirac operator $\mathcal{D}$ by

$\mathcal{D} \equiv-\frac{i}{2} \gamma_{\alpha} \gamma_{\beta} M^{\alpha \beta}+2=-\not x \not \partial^{T}+2$,

then $(2.11)$ can be written as follows:

$(i \mathcal{D}-v)(i \mathcal{D}+v) \psi(x)=0$.

This relation is similar to the standard relation of the spinor field in flat space, $(i \not \partial+m)(i \not \partial-m) \psi(x)=0$. The first-order field equation for a field of spin- $\frac{1}{2}$ in $\mathrm{dS}$ space becomes

$(i \mathcal{D}+v) \psi(x)=0, \quad$ where $v \neq 0 \in \mathbb{R}$.

The massless case in the de Sitter space belongs to the discrete series and the eigenvalue of the second-order Casimir operator is given by

$\left\langle Q_{s}^{(1)}\right\rangle=2\left(1-s^{2}\right) \mathrm{Il}$.

Plugging this value for $s=\frac{1}{2}$ in (2.7) and in terms of the $\mathcal{D}$ operator, one obtains

$i \mathcal{D} \psi(x)=0$, or equivalently $\left(Q_{0}-2\right) \psi=0$.

Similarly for spin- $\frac{3}{2}$ field equation in $\mathrm{dS}$ space, two types of UIRs of the $\mathrm{dS}$ group are characterized, the principal and discrete series:

(i) The unitary irreducible representations $U_{\frac{3}{2}, v}$ of the principal series,

$$
\left\langle Q_{\frac{3}{2}}^{(1)}\right\rangle=\left(v^{2}-\frac{3}{2}\right), \quad v \in \mathbb{R} \quad v>\frac{3}{2},
$$

note that $U_{\frac{3}{2}, v}$ and $U_{\frac{3}{2},-v}$ are equivalent this kind of the representation belong to the massive case.

(ii) We have the unitary irreducible representations $\Pi_{\frac{3}{2}, \frac{3}{2}}^{ \pm}$of the discrete series, and

$\left\langle Q_{\frac{3}{2}}^{(1)}\right\rangle=-\frac{5}{2}$,

the sign \pm stands for the helicity.

The massless spin- $\frac{3}{2}$ field in $\mathrm{dS}$ space becomes $[14,15]$

$\left(Q_{\frac{3}{2}}^{(1)}+\frac{5}{2}\right) \Psi_{\alpha}(x)=0$,

where

$$
\begin{aligned}
Q_{\frac{3}{2}}^{(1)} \Psi_{\alpha}(x)= & \left(-\frac{1}{2} M_{\alpha \beta} M^{\alpha \beta}+\frac{i}{2} \gamma_{\alpha} \gamma_{\beta} M^{\alpha \beta}-\frac{11}{2}\right) \Psi_{\alpha}(x) \\
& -2 \partial_{\alpha} x \cdot \Psi(x)+2 x_{\alpha} \partial \cdot \Psi(x)+\gamma_{\alpha} \gamma \cdot \Psi(x) .
\end{aligned}
$$

Similar to the massless vector-spinor fields equations in Minkowski space, the solutions of this field equation possess a singularity due to the divergencelessness condition $\left(\partial^{T} \cdot \psi=0\right)$. Then the gauge invariant field equation is [15]

$$
\left(Q_{\frac{3}{2}}^{(1)}+\frac{5}{2}\right) \Psi_{\alpha}(x)-\mathcal{D}_{\alpha}^{\left(\frac{3}{2}\right)} \partial^{T} \cdot \Psi(x)=0,
$$

where $\mathcal{D}_{\alpha}^{\left(\frac{3}{2}\right)} \equiv-\partial_{\alpha}^{T}-\gamma_{\alpha}^{T} \not x$. This equation is invariant under the following gauge transformation:

$\Psi_{\alpha}(x) \rightarrow \Psi_{\alpha}^{\prime}(x)=\Psi_{\alpha}(x)+\mathcal{D}_{\alpha}^{\left(\frac{3}{2}\right)} \zeta$

where $\zeta$ is an arbitrary spinor field. In order to fix the gauge, $c$ is introduced and then one has

$$
\left(Q_{\frac{3}{2}}^{(1)}+\frac{5}{2}\right) \Psi_{\alpha}(x)-c \mathcal{D}_{\alpha}^{\left(\frac{3}{2}\right)} \partial^{T} \cdot \Psi(x)=0 .
$$

Similar to the spin-1/2 case, one can write the first-order field equation as

$$
\not x \not \partial{ }^{T} \Psi_{\alpha}(x)-3 \Psi_{\alpha}(x)-x_{\alpha} \not x \not \Psi-\partial_{\alpha}^{T} \not x \not \Psi=0 .
$$

This equation is invariant under the gauge transformation $\Psi_{\alpha} \rightarrow \Psi_{\alpha}^{\prime}=\Psi_{\alpha}+\mathcal{D}_{\alpha}^{\left(\frac{3}{2}\right)} \zeta$. There exists another first-order field equation [8]:

$\not x \not \partial^{T} \Psi_{\alpha}(x)-\Psi_{\alpha}(x)-x_{\alpha} \not x \not \Psi+\mathcal{D}_{\alpha}^{\left(\frac{3}{2}\right)} \not x \not \Psi=0$, 
which is invariant under the gauge transformation $\Psi_{\alpha} \rightarrow$ $\Psi_{\alpha}^{\prime}=\Psi_{\alpha}+\partial_{\alpha}^{T} \zeta$. It is worth to mention that (2.24) appears in the conformal invariant field equation.

\section{Conformal invariance and Dirac's six-cone formalism}

Massless field equations are expected to be conformally invariant (CI). A trivial example is the Maxwell equations; 1909 Cunningham and Bateman showed that these equations are covariant under the larger 15-parameter conformal group $S O(2,4)$ as well as 10-parameter Poincaré group [34]. Fields with spin $s \geq 1$ are invariant under the gauge transformation as well. In 1936 Dirac used a manifestly conformally covariant formulation namely, the conformal space notation, to write down wave equations in Minkowski space [35]. The conformal group acts non-linearly on Minkowski coordinates, Dirac used coordinates which the conformal group acts linearly on them. This actually reassembles the conformal space and the theory is defined on a $d+1$-dimensional hypercone (hereafter named Dirac's six-cone) or equivalently, in a $d+2$-dimensional conformal space. Within this formalism, he obtained scalar, spinor and vector conformally invariant fields in $d=4$ flat space-time. This theory developed in some papers $([36,37]$ and references therein). The generalization to dS space was done in $[38,39]$ to obtain CI field equations for scalar, vector and symmetric rank-2 tensor fields. Here, we use this approach to study the spinor fields $\left(s=\frac{1}{2}\right.$ and $\left.\frac{3}{2}\right)$ in the de Sitter space. First let us recall this method briefly.

\subsection{Dirac's six-cone}

Basically, the special conformal transformation acts nonlinearly on four-dimensional coordinates. In conformal space Dirac proposed the coordinates $u^{a}$, where $S O(2,4)$ acts linearly on them. Dirac's six-cone is then defined as a fivedimensional hyper-surface in $\mathbb{R}^{6}$ satisfying the following constraint:

$u^{2}=\eta_{a b} u^{a} u^{b}=u_{0}^{2}-\vec{u}^{2}+u_{5}^{2}=0, \quad a, b=0,1, \ldots, 5$,

this is obviously invariant under the conformal transformation. A given operator say as $\hat{A}$ is said to be intrinsic if it satisfies

$\hat{A} u^{2} \phi=u^{2} \hat{A} \phi$,

where $\phi$ is a function in $\mathbb{R}^{6}$. One should write all the wave equations, subsidiary conditions and etc., in terms of operators that are defined intrinsically on the cone. The following CI system which is defined on the cone is well established aiming to this goal ${ }^{2}[38]$ :

$\left\{\begin{array}{l}\left(\partial_{a} \partial^{a}\right)^{n} \Phi=0, \\ \hat{N}_{5} \Phi=(n-2) \Phi\end{array}\right.$

where the powers of d'Alembertian $\left(\partial_{a} \partial^{a}\right)^{n}$ act intrinsically on fields of conformal degree $(n-2)$, and $\Phi$ is a tensor or spinor field of a definite rank and symmetry. The conformaldegree operator $\hat{N}_{5}$ is given by

$\hat{N}_{5} \equiv u^{a} \partial_{a}$.

One can add the following CI conditions to restrict the solution space:

(i) transversality: $u_{a} \Phi^{a b \ldots}=0$,

(ii) divergencelessness: $\nabla_{a} \Phi^{a b \cdots}=0$,

(iii) tracelessness: $\Phi_{a b \ldots}^{a}=0$,

(iv) for tensor-spinor field: $\gamma_{a} \Phi^{a b \cdots}=0$.

In conformal coordinate $u$, the definition of $\nabla$ is given by [41]

$\nabla_{a} \equiv u_{a} \partial_{b} \partial^{b}-\left(2 \hat{N}_{5}+4\right) \partial_{a}$.

The quantities which are evaluated on the cone should be projected to $4+1$ de Sitter space, first, one needs a relation between the coordinates

$\left\{\begin{array}{l}x^{\alpha}=\left(u^{5}\right)^{-1} u^{\alpha} \\ x^{5}=u^{5}\end{array}\right.$

therefore, the intrinsic operators turn to $[38,41]$ :

$\hat{N}_{5}=x_{5} \frac{\partial}{\partial x_{5}}$,

$\left(\partial_{a} \partial^{a}\right)^{n}=-x_{5}^{-2 n} \prod_{j=1}^{n}\left[Q_{0}+(j+1)(j-2)\right]$,

$\nabla_{\alpha}=-x_{5}^{-1}\left[x_{\alpha}\left[Q_{0}-\hat{N}_{5}\left(\hat{N}_{5}-1\right)\right]+2 \partial_{\alpha}^{T}\left(\hat{N}_{5}+1\right)\right]$,

where $Q_{0}$ is the Casimir operator in the de Sitter space. First, one should write the equations in $u$ coordinates where the conformal invariance is manifest (3.2). Then with the help of the above mentioned relations, the obtained equations are related to the embedding space ones. Finally, the desired de Sitter relations can be obtained via the projection.

\footnotetext{
${ }^{2}$ In fact, this approach to conformal symmetry leads to the best path to exploit the physical symmetry and it provides a rather simple way to write the conformally invariant field equations. Moreover, it is important to mention that on the cone $u^{2}=0$, the second-order Casimir operator of the conformal group, $\mathcal{Q}_{2}$, is not a suitable operator to obtain the $\mathrm{CI}$ wave equations. Because it is proved on the cone $u^{2}=0$, it reduces to a constant, consequently, this operator cannot lead to the wave equations on the cone. The well-defined operators exist only in exceptional cases. For tensor fields of degree $-1,0,1, \ldots$, the intrinsic wave operators are $\partial^{2},\left(\partial^{2}\right)^{2},\left(\partial^{2}\right)^{3}, \ldots$, respectively. This method previously was established for $3+2$-de Sitter linear gravity in Ref. [40].
} 
This approach provides a simple way to study the conformal invariance in the de Sitter space.

\subsection{Conformally invariant spinor field equations}

Spinor field $s=\frac{1}{2}$ : The simplest conformally invariant system is obtained by setting $n=1$ and $\psi \equiv x_{5} \Phi$ in (3.2) which after making use of (3.5) and in language of the Casimir operator of the $\mathrm{dS}$ group, it turns to

$\left(Q_{0}-2\right) \psi(x)=0$.

This equation is obviously conformally invariant, and $\psi(x)$ stands for a massless conformally spinor field in dS space (see Eq. 2.16). After making use of (2.11), the first-order field equation in this case, becomes

$\left(\not x \not \partial^{T}-2\right) \psi(x)=0$.

Indeed, the field $\psi(x)$, associates with the UIR of the $\mathrm{dS}$ group $\Pi_{\frac{1}{2}, \frac{1}{2}}^{ \pm}$and propagates on the dS light cone.

Vector-spinor $s=\frac{3}{2}$ : In this case one should classify the degrees of freedom of a vector-spinor field on the cone in terms of the dS fields, this can be done as below

$\Psi_{\alpha}=x_{5}\left(\Phi_{\alpha}+x_{\alpha} x \cdot \Phi\right), \quad \mathcal{K}_{1}=x_{5} \Phi_{5}, \quad \mathcal{K}_{2}=x_{5} x \cdot \Phi$,

where $\mathcal{K}_{1}$ and $\mathcal{K}_{2}$ are two spinor fields and $\Psi_{\alpha}$ is a vectorspinor field. Note that $x^{\alpha} \Psi_{\alpha}=0$ indicates that $\Psi_{\alpha}$ lives on a $\mathrm{dS}$ hyperboloid. Taking CI field equations by choosing $n=1$ in (3.2), one obtains

$\left(Q_{0}-2\right) \Phi_{\alpha}=0$,

where $\Phi_{\alpha}$ is a spin- $\frac{3}{2}$ field on the cone. After doing some tedious but straightforward calculation, which partially is given in Appendix C, the following CI system of field equations is obtained:

$$
\left\{\begin{array}{l}
\left(Q_{0}-2\right) \mathcal{K}_{1}=0 \\
\left(Q_{0}-2\right) \mathcal{K}_{2}=0 \\
\left(Q_{0}-2\right) \Psi_{\alpha}+2 x_{\alpha} \partial^{T} \cdot \Psi+\partial_{\alpha}^{T} \partial^{T} \cdot \Psi=0
\end{array}\right.
$$

which indicates the following: $\mathcal{K}_{1}$ and $\mathcal{K}_{2}$ are both CI massless spinor fields [38]. By using the transversality condition on the cone, $\left(u_{a} \Phi^{a b \ldots}=0=u_{5} \Phi^{5}+u_{\alpha} \Phi^{\alpha}\right)$, and (3.9), we obtain (see Appendix C)

$$
\left(Q_{0}-2\right) \partial^{T} . \Psi=0 .
$$

However, one can use (2.19) to write the third line of (3.10) as

$$
\left(Q_{\frac{3}{2}}^{(1)}-\not x \not \partial^{T}+\frac{7}{2}\right) \Psi_{\alpha}+\partial_{\alpha}^{T} \partial^{T} \cdot \Psi-\gamma_{\alpha} \not \Psi=0 .
$$

Using $(2.18,2.24)$ the above field equation can be rewritten as

$$
\begin{aligned}
& \left(Q_{\frac{3}{2}}^{(1)}+\frac{5}{2}\right) \Psi_{\alpha}-\left(\not x \partial^{T} \Psi_{\alpha}-\Psi_{\alpha}-x_{\alpha} \not x \not \Psi\right. \\
& \left.-\partial_{\alpha}^{T} \partial^{T} \cdot \Psi+\gamma_{\alpha}^{T} \not \Psi\right)=0 .
\end{aligned}
$$

As previously mentioned, the fields should be projected to the de Sitter space, the transverse projection implies the transversality of fields, $x \cdot \Psi=0$, so that from the homogeneity condition, one obtains $x^{\alpha} x \cdot \partial \Psi_{\alpha}=0$. These two conditions impose the following constrains on the projected fields: $x \cdot \Phi=0=\Phi^{5}$, and consequently $\Psi_{\alpha}=\Phi_{\alpha}$. In Appendix $C$, it is shown that the CI divergencelessness condition on the cone, namely $\nabla_{a} \Phi^{a}=0$, results in $\partial^{T}{ }_{\alpha} \Psi^{\alpha}=0=\partial^{T}{ }_{\alpha} \Phi^{\alpha}$, which indicates the divergenceless fields are only mapped from the cone on dS hyperboloid.

For simplicity and irreducibility of a vector-spinor field representation, the CI condition $\gamma^{a} \Phi_{a}=0$ on the cone is imposed, this leads to $\gamma^{\beta} \Psi_{\beta}=\not \Psi=0$, which is the conformally invariant condition on the de Sitter hyperboloid. Imposing this condition and irreducibility (see 2.18), one obtains the following first- and second-order CI field equations:

$\left(\not x \not \partial^{T}-1\right) \Psi_{\alpha}(x)=0, \quad$ and $\quad\left(Q_{\frac{3}{2}}^{(1)}+\frac{5}{2}\right) \Psi_{\alpha}=0$.

In this case $\Psi_{\alpha}$ associates with the UIR of the dS group, namely $\Pi_{\frac{3}{2}, \frac{3}{2}}^{ \pm}$, and note that it propagates on the dS light cone. In the following sections, we find the solution and also obtain the two-point function of this vector-spinor field.

\section{Solutions of the conformally invariant wave equations}

A general solution of the field equation (3.14) can be written in terms of the three spinor fields $\psi_{1}, \psi_{2}$, and $\psi_{3}$ as follows:

$\Psi_{\alpha}(x)=Z_{\alpha}^{T} \psi_{1}+\mathcal{D}_{\alpha}^{\left(\frac{3}{2}\right)} \psi_{2}+\gamma_{\alpha}^{T} \psi_{3}$,

where $Z^{\alpha}$ is an arbitrary five-component constant vector field and $x \cdot Z^{T}=0$. Now we should identify the introduced spinor fields $\psi_{1}, \psi_{2}$ and $\psi_{3}$. If one demands that $\Psi_{\alpha}$ satisfies the second-order field equation (3.14), then the spinor fields $\psi_{1}, \psi_{2}$ and $\psi_{3}$ obey the following equations:

$\left(Q_{0}+\not x \not \partial^{T}-3\right) \psi_{1}=0$,

$2(x \cdot Z) \psi_{1}+\left(Q_{0}+\not x \partial^{T}\right) \psi_{2}=0$, 
On the other hand, $\Psi_{\alpha}$ must satisfy the first-order field equation (3.14); therefore one obtains

$$
\begin{aligned}
& \left(\not x \not^{T}-1\right) \psi_{1}=0, \\
& (\not x-2) \psi_{2}-2 \not x \psi_{3}=0, \\
& \not x \not \partial^{T} \psi_{3}-(4 \not x+1) \psi_{2}-\not x \cdot Z \psi_{1}=0 .
\end{aligned}
$$

The second line of equation (4.3) results in

$\psi_{2}=\frac{2}{5}(1-2 \not x) \psi_{3}, \quad$ or $\quad \psi_{3}=\frac{1}{2}(1+2 \not x) \psi_{2}$.

From (4.2) and (4.3), $\psi_{1}$ satisfies the following first- and second-order field equations:

$\left(Q_{0}-2\right) \psi_{1}=0, \quad\left(\not x \not \partial^{T}-1\right) \psi_{1}=0$,

that indicates it can be regarded as a massless conformally coupled spinor field with homogeneity degree of $\sigma=-1$ and $-2[32,33]$. On the other hand, the homogeneity consideration reveals that the degrees of homogeneity of $\psi_{3}$ and $\psi_{1}$ are equal, note that all the sentences in (4.1) have the same degrees of homogeneity and also the degrees of homogeneity $Z_{\alpha}^{T}$ and $\gamma_{\alpha}^{T}$ are zero.

Now let us multiply $\not^{T}$ from the left on equations (4.3); after doing some calculations, this yields

$$
\begin{aligned}
& Q_{0} \psi_{2}=\frac{2}{5}(1-2 \not x) \psi_{2}+2\left(3 x \cdot Z-\not x \not Z^{T}\right) \psi_{1}, \\
& Q_{0} \psi_{3}=-\frac{2}{5}(2 \not x+7) \psi_{3}+\left(3 x \cdot Z-\not x \not Z^{T}\right) \psi_{1} .
\end{aligned}
$$

Inserting these results in equations (4.2), and after making use of (4.4), one can write $\psi_{2}$ and $\psi_{3}$ in terms of $\psi_{1}$ as follows:

$$
\begin{aligned}
& \psi_{2}=\frac{1}{2}(1+3 \not x)((1+3 \not x) x \cdot Z+\not Z) \psi_{1}, \\
& \psi_{3}=-\frac{5}{4}(1-\not x)((1+3 \not x) x \cdot Z+\not Z) \psi_{1} .
\end{aligned}
$$

Using the divergencelessness condition and the above relations, one obtains

$$
\left(Z \cdot \partial^{T}+3 x \cdot Z\right) \psi_{1}+\frac{1}{2}(\not x-3)((1+3 \not x) x \cdot Z+\not Z) \psi_{1}=0,
$$

and making use of $\not \Psi=0$ leads one to write

$$
\not^{T} \psi_{1}-\left(4 \not \not x+\not \partial^{T}\right) \psi_{2}+4 \psi_{3}=0,
$$

and in terms of $\psi_{1}$, this equation becomes

$$
\begin{aligned}
& Z^{T} \psi_{1}+2(\not x+5)[(1+3 \not x) x \cdot Z+\not Z] \psi_{1} \\
& -\frac{2}{5}(1+2 \not x) x \cdot Z \psi_{1}=0 .
\end{aligned}
$$

Actually what we have obtained is this: by dealing with $\psi_{1}$, the other two fields will be established as well. Consequently by gathering all the results, $\Psi$ can be written as follows:

$\Psi_{\alpha}(x)=\mathbf{D}_{\alpha}\left(x, \partial^{T}, Z\right) \psi_{1}$, where we have defined

$$
\begin{aligned}
& \mathbf{D}_{\alpha}\left(x, \partial^{T}, Z\right) \equiv Z_{\alpha}^{T} \\
& +\left[\frac{1}{2} \mathcal{D}_{\alpha}^{\left(\frac{3}{2}\right)}(1+3 \not x)-\frac{5}{4} \gamma_{\alpha}^{T}(1-\not x)\right]((1+3 \not x) x \cdot Z+\not Z) .
\end{aligned}
$$

In this formalism a given spin- $\frac{3}{2}$ field could be constructed from the multiplication of the polarization vector $\mathbf{D}_{\alpha}$ or $Z_{\alpha}$ ( 5 degrees of freedom) with the spinor field $\psi_{1}$ (2 degrees of freedom), which appears naturally 10 polarization states. After making use of (4.10) and (4.11), the degrees of freedom are indeed reduced to the usual four polarization states $m_{\frac{3}{2}}=$ $\frac{3}{2}, \frac{1}{2},-\frac{1}{2}$ and $-\frac{3}{2}$, where two of them are the physical states $\pm \frac{3}{2}[8]$.

Now, $\psi_{1}$ should be identified. Making use of the relation between the $s=1 / 2$ and spin-zero Casimir operators, (4.5) can be written as

$$
\left(Q_{\frac{1}{2}}^{(1)}-\frac{1}{2}\right) \psi_{1}(x)=0 .
$$

This means that $\psi_{1}$ and its related two-point functions can in fact be extracted from a massive spinor field in the principal series representation given by (2.8) by setting $v=-i$. Massive spinor field and its two-point functions has already been studied in $[32,33]$. Therefore, the solutions of $(4.5)$ are found to be

$\psi_{1}(x)=(x \cdot \xi)^{-2} \mathcal{V}(x, \xi)$,

$\psi_{1}^{\prime}(x)=(x \cdot \xi)^{-1} \mathcal{U}(\xi)$,

$\mathcal{V}(x, \xi) \equiv \frac{x \xi}{x \cdot \xi} \mathcal{V}(\xi)$, these solutions are the de Sitter-Dirac plane waves. $\xi$ is a vector that lives in the positive sheet of the light cone, i.e.,

$\xi \in \mathbb{C}^{+}=\left\{\xi ; \eta_{\alpha \beta} \xi^{\alpha} \xi^{\beta}=\left(\xi^{0}\right)^{2}-\vec{\xi} \cdot \vec{\xi}-\left(\xi^{4}\right)^{2}=0, \xi^{0}>0\right\}$.

$\xi$ plays the role of the energy momentum in the null curvature limit. Two spinors $\mathcal{V}(\xi)$ and $\mathcal{U}(\xi)$ are given by [32,33]

$\mathcal{U}^{i}(\xi)=\frac{\xi^{0}-\vec{\xi} \cdot \vec{\gamma} \gamma^{0}+1}{\sqrt{2\left(\xi^{0}+1\right)}} \mathcal{U}^{i}\left(\stackrel{o}{\xi}_{+}\right)$,
$\mathcal{V}^{i}(\xi)=\frac{1}{\sqrt{2\left(\xi^{0}+1\right)}} \mathcal{U}^{i}\left(\stackrel{o}{\xi}_{-}\right), \quad i=1,2$,

where

$\mathcal{U}_{1}\left(\xi_{+}^{o}\right)=\frac{1}{\sqrt{2}}\left(\begin{array}{l}\chi \\ \chi\end{array}\right), \quad \mathcal{U}_{2}\left(\xi_{+}^{o}\right)=\frac{1}{\sqrt{2}}\left(\begin{array}{l}\chi^{\prime} \\ \chi^{\prime}\end{array}\right)$,

$\mathcal{U}_{1}\left(\xi_{-}^{o}\right)=\frac{1}{\sqrt{2}}\left(\begin{array}{c}\chi \\ -\chi\end{array}\right), \quad \mathcal{U}_{2}\left(\xi_{-}^{o}\right)=\frac{1}{\sqrt{2}}\left(\begin{array}{c}\chi^{\prime} \\ -\chi^{\prime}\end{array}\right)$,

in which $\chi=\left(\begin{array}{l}1 \\ 0\end{array}\right), \chi^{\prime}=\left(\begin{array}{l}0 \\ 1\end{array}\right)$ and $\xi=\stackrel{o}{\xi}_{ \pm} \equiv(1, \overrightarrow{0}, \pm 1)$. 
Therefore we have two solutions for $\Psi_{\alpha}(x)$, which are as follows:

$$
\begin{aligned}
\Psi_{1 \alpha}(x) & =\mathbf{D}_{\alpha}\left(x, \partial^{T}, Z\right) \mathcal{V}(x, \xi)(x \cdot \xi)^{-2} \\
& \equiv \mathcal{V}_{\alpha}(x, \xi, Z)(x \cdot \xi)^{-2}
\end{aligned}
$$

and

$$
\begin{aligned}
\Psi_{2 \alpha}(x) & =\mathbf{D}_{\alpha}\left(x, \partial^{T}, Z\right) \mathcal{U}(\xi)(x \cdot \xi)^{-1} \\
& \equiv \mathcal{U}_{\alpha}(x, \xi, Z)(x \cdot \xi)^{-1}
\end{aligned}
$$

After doing some calculation and taking the derivative, the explicit forms of $\mathcal{U}_{\alpha}$ and $\mathcal{V}_{\alpha}$ are obtained in terms of $x, \xi$, and $Z$.

\section{Two-point function in ambient space formalism}

In this section, we deal with conformally invariant two-point functions of a massless spin- $\frac{3}{2}$ field. They are found in terms of the spinor (spin-1/2) two-point function. The two-point function of the massless spin- $\frac{3}{2}$ field is given by

$S_{\alpha \alpha^{\prime}}^{i \bar{j}}\left(x, x^{\prime}\right)=\left\langle\Omega\left|\Psi_{\alpha}^{i}(x) \bar{\Psi}_{\alpha^{\prime}}^{\bar{j}}\left(x^{\prime}\right)\right| \Omega\right\rangle$,

where $x, x^{\prime} \in X_{H}$, and $\mid \Omega>$ stands for the vacuum state. Let us use a similar procedure to the previous section and write the desired two-point function in terms of three spinor twopoint functions and impose some conditions to write down the two of them as a function of the third one. By using (4.1) and (5.1), the following form of the two-point function is proposed:

$$
\begin{aligned}
\mathcal{S}_{\alpha \alpha^{\prime}}\left(x, x^{\prime}\right)= & \theta_{\alpha} \cdot \theta_{\alpha^{\prime}}^{\prime} \mathcal{S}_{1}\left(x, x^{\prime}\right)-\mathcal{D}_{\alpha}^{\left(\frac{3}{2}\right)} \mathcal{S}_{2}\left(x, x^{\prime}\right) \gamma^{4} \overleftarrow{\mathcal{D}}_{\alpha^{\prime}}^{\prime\left(\frac{3}{2}\right)} \gamma^{4} \\
& -\gamma_{\alpha}^{T} \mathcal{S}_{3}\left(x, x^{\prime}\right) \gamma^{4} \gamma_{\alpha^{\prime}}^{\prime T} \gamma^{4}
\end{aligned}
$$

where the prime operators act only on $x^{\prime}$. This two-point function must satisfy the CI system of the field equations (3.14). If one demands that $\mathcal{S}_{\alpha \alpha^{\prime}}\left(x, x^{\prime}\right)$ satisfies the secondorder field equation and using the identities of Appendix B, the following relations are obtained:

$$
\begin{aligned}
& \left(Q_{0}+\not x \not \partial^{T}-3\right) \mathcal{S}_{1}\left(x, x^{\prime}\right)=0, \\
& 2\left(x \cdot \theta_{\alpha^{\prime}}^{\prime}\right) \mathcal{S}_{1}\left(x, x^{\prime}\right) \\
& \quad+\left(Q_{0}+\not x \not \partial^{T}\right) \mathcal{S}_{2}\left(x, x^{\prime}\right) \gamma^{4} \overleftarrow{\mathcal{D}}_{\alpha^{\prime}}^{\prime\left(\frac{3}{2}\right)} \gamma^{4}=0, \\
& {\left[-3 \not x\left(x \cdot \theta_{\alpha^{\prime}}^{\prime}\right)+\left(\theta_{\alpha^{\prime}}^{\prime} \cdot \gamma_{\alpha}^{T}\right)\right] \mathcal{S}_{1}\left(x, x^{\prime}\right)} \\
& \quad-\left(Q_{0}+\not x \not \partial^{T}\right) \mathcal{S}_{3}\left(x, x^{\prime}\right) \gamma^{4} \gamma_{\alpha^{\prime}}^{\prime T} \gamma^{4}=0 .
\end{aligned}
$$

On the other hand, $\mathcal{S}_{\alpha \alpha^{\prime}}\left(x, x^{\prime}\right)$ must satisfy the first-order equation, and then one obtains

$$
\begin{aligned}
& \left(\not x \not \partial^{T}-1\right) \mathcal{S}_{1}\left(x, x^{\prime}\right)=0 \\
& (\not x-2) \mathcal{S}_{2}\left(x, x^{\prime}\right) \gamma^{4} \overleftarrow{\mathcal{D}}_{\alpha^{\prime}}^{\frac{3}{2}} \gamma^{4}-2 \not x \mathcal{S}_{3}\left(x, x^{\prime}\right) \gamma^{4} \gamma_{\alpha^{\prime}}^{\prime T} \gamma^{4}=0 \\
& \not x \not \partial^{T} \mathcal{S}_{3}\left(x, x^{\prime}\right) \gamma^{4} \gamma_{\alpha^{\prime}}^{\prime T} \gamma^{4}-(\not x+4) \mathcal{S}_{2}\left(x, x^{\prime}\right) \gamma^{4} \overleftarrow{\mathcal{D}}_{\alpha^{\prime}}^{\prime \frac{3}{2}} \gamma^{4} \\
& \quad+x_{\alpha^{\prime}} \not x \mathcal{S}_{1}\left(x, x^{\prime}\right)=0
\end{aligned}
$$

We can write $\mathcal{S}_{2}\left(x, x^{\prime}\right)$ in terms of $\mathcal{S}_{3}\left(x, x^{\prime}\right)$ and vice versa by using the second equation (5.6) as follows:

$\mathcal{S}_{2}\left(x, x^{\prime}\right) \gamma^{4} \overleftarrow{\mathcal{D}}_{\alpha^{\prime}}^{\prime \frac{3}{2}} \gamma^{4}=\frac{2}{5}(1-2 \not x) \mathcal{S}_{3}\left(x, x^{\prime}\right) \gamma^{4} \gamma_{\alpha^{\prime}}^{\prime T} \gamma^{4}$

$\mathcal{S}_{3}\left(x, x^{\prime}\right) \gamma^{4} \gamma_{\alpha^{\prime}}^{\prime T} \gamma^{4}=\frac{1}{2}(1+2 \not x) \mathcal{S}_{2}\left(x, x^{\prime}\right) \gamma^{4} \overleftarrow{\mathcal{D}}_{\alpha^{\prime}}^{\prime \frac{3}{2}} \gamma^{4}$

The first-order equation of two-point function can be written as

$$
\begin{aligned}
& \left(\not x \not \partial^{T}-1\right) \mathcal{S}_{1}\left(x, x^{\prime}\right)=0 \\
& (\not x+2) \not \partial^{T} \mathcal{S}_{2}\left(x, x^{\prime}\right) \gamma^{4} \overleftarrow{\mathcal{D}}_{\alpha^{\prime}}^{\frac{3}{2}} \gamma^{4} \\
& +2(\not x-8) \mathcal{S}_{2}\left(x, x^{\prime}\right) \gamma^{4} \overleftarrow{\mathcal{D}}_{\alpha^{\prime}}^{\prime \frac{3}{2}} \gamma^{4}+2 x_{\alpha^{\prime}} \not x \mathcal{S}_{1}\left(x, x^{\prime}\right)=0 \\
& \not x \not \partial^{T} \mathcal{S}_{3}\left(x, x^{\prime}\right) \gamma^{4} \gamma_{\alpha^{\prime}}^{\prime T} \gamma^{4}-\frac{2}{5}(6-7 \not x) \mathcal{S}_{3}\left(x, x^{\prime}\right) \gamma^{4} \gamma_{\alpha^{\prime}}^{\prime T} \gamma^{4} \\
& +x_{\alpha^{\prime}} \not x \mathcal{S}_{1}\left(x, x^{\prime}\right)=0 .
\end{aligned}
$$

The equation governing $\mathcal{S}_{1}$ can be deduced from (5.3) and the first equation of (5.6) as

$\left(Q_{0}-2\right) S_{1}\left(x, x^{\prime}\right)=0, \quad\left(\not x \not \partial{ }^{T}-1\right) \mathcal{S}_{1}\left(x, x^{\prime}\right)=0$.

In Ref. [32,33], the solutions have been obtained; here, we only quote the result:

$S_{1}\left(x, x^{\prime}\right)=\frac{i}{2(4 \pi)^{2}}\left[P_{-3}^{(7)}\left(x \cdot x^{\prime}\right) \not x-3 P_{-1}^{(7)}\left(x \cdot x^{\prime}\right) \not x^{\prime}\right] \gamma^{4}$,

$P_{\sigma}^{(7)}$ are the generalized Legendre functions (see Appendix D). Similar to the previous section, by acting $\not{ }^{T}$ on the first-order equation (5.8), one finds the second-order equation for $\mathcal{S}_{2}$ and $\mathcal{S}_{3}$ as follows:

$$
\begin{aligned}
& Q_{0} \mathcal{S}_{2}\left(x, x^{\prime}\right) \gamma^{4} \overleftarrow{\mathcal{D}}_{\alpha^{\prime}}^{\prime \frac{3}{2}} \gamma^{4}=-2(3 \not x+20) \mathcal{S}_{2}\left(x, x^{\prime}\right) \gamma^{4} \overleftarrow{\mathcal{D}}_{\alpha^{\prime}}^{\prime \frac{3}{2}} \gamma^{4} \\
& +\frac{2}{5}\left[2 x_{\alpha^{\prime}}(1+7 \not x)+(1-2 \not x) \gamma_{\alpha^{\prime}}^{T}\right] \mathcal{S}_{1}\left(x, x^{\prime}\right)
\end{aligned}
$$

$$
\begin{aligned}
& Q_{0} \mathcal{S}_{3}\left(x, x^{\prime}\right) \gamma^{4} \gamma_{\alpha^{\prime}}^{\prime T} \gamma^{4}=\frac{2}{5}(7 \not x-16) \mathcal{S}_{3}\left(x, x^{\prime}\right) \gamma^{4} \gamma_{\alpha^{\prime}}^{\prime T} \gamma^{4} \\
& +\left[\frac{2}{5} x_{\alpha^{\prime}}(6 \not x-7)+\gamma_{\alpha^{\prime}}^{T}\right] \mathcal{S}_{1}\left(x, x^{\prime}\right)
\end{aligned}
$$


After making use of the above relations in (5.5), one can write $\mathcal{S}_{2}$ and $\mathcal{S}_{3}$ in terms of $\mathcal{S}_{1}$ as follows:

$$
\begin{aligned}
\mathcal{S}_{2}\left(x, x^{\prime}\right) \gamma^{4} \overleftarrow{\mathcal{D}}_{\alpha^{\prime}}^{\prime \frac{3}{2}} \gamma^{4} \\
=-\frac{1}{10}(1-2 \not x)\left[-3 \not x\left(x \cdot \theta_{\alpha^{\prime}}^{\prime}\right)+\left(\theta_{\alpha^{\prime}}^{\prime} \cdot \gamma_{\alpha}^{T}\right)\right. \\
\left.\quad-\frac{1}{5} x_{\alpha^{\prime}}(7 \not x-14)-\gamma_{\alpha^{\prime}}^{T}\right] \mathcal{S}_{1}\left(x, x^{\prime}\right),
\end{aligned}
$$

$$
\begin{aligned}
\mathcal{S}_{3}\left(x, x^{\prime}\right) \gamma^{4} \gamma_{\alpha^{\prime}}^{\prime T} \gamma^{4} & \\
= & -\frac{1}{4}\left[-3 \not x\left(x \cdot \theta_{\alpha^{\prime}}^{\prime}\right)+\left(\theta_{\alpha^{\prime}}^{\prime} \cdot \gamma_{\alpha}^{T}\right)\right. \\
& \left.\quad-\frac{1}{5} x_{\alpha^{\prime}}(7 \not x-14)-\gamma_{\alpha^{\prime}}^{T}\right] \mathcal{S}_{1}\left(x, x^{\prime}\right) .
\end{aligned}
$$

Finally the two-point function takes the following form in terms of $\mathcal{S}_{1}$ :

$\mathcal{S}_{\alpha \alpha^{\prime}}\left(x, x^{\prime}\right)=\mathbf{D}_{\alpha \alpha^{\prime}}\left(x, \partial^{T} ; x^{\prime}, \partial^{\prime T}\right) \mathcal{S}_{1}\left(x, x^{\prime}\right)$,

where we have

$$
\begin{aligned}
& \mathbf{D}_{\alpha \alpha^{\prime}}\left(x, \partial^{T} ; x^{\prime}, \partial^{\prime T}\right) \\
& \quad=\theta_{\alpha} \cdot \theta_{\alpha^{\prime}}^{\prime}+\left(\frac{1}{10} \mathcal{D}_{\alpha}^{\left(\frac{3}{2}\right)}(1-2 \not x)+\frac{1}{4} \gamma_{\alpha}^{T}\right) \\
& \quad \times\left[-3 \not x\left(x \cdot \theta_{\alpha^{\prime}}^{\prime}\right)+\left(\theta_{\alpha^{\prime}}^{\prime} \cdot \gamma_{\alpha}^{T}\right)-\frac{1}{5} x_{\alpha^{\prime}}(7 \not x-14)-\gamma_{\alpha^{\prime}}^{T}\right] .
\end{aligned}
$$

This two-point function is conformally invariant.

\section{Two-point functions in intrinsic coordinates}

The two-point function in intrinsic coordinates of de Sitter space can indeed be obtained by projecting $\mathcal{S}_{\alpha, \alpha^{\prime}}$. To do this let us recall briefly the de Sitter-Dirac equation in embedding and intrinsic spaces. In embedding space $y^{\alpha} \equiv\left(y^{\mu}, y^{4}=\right.$ $\left.H^{-1}\right)$ is introduced with the following relation with $x^{\alpha}$ [42]:

$x^{\alpha}=\left(H y^{4}\right) f^{\alpha}\left(y^{0}, y^{1}, y^{2}, y^{3}\right)$,

where the arbitrary function $f^{\alpha}$ satisfying $f^{\alpha} f_{\alpha}=-H^{-2}$. The five matrices $\beta^{\alpha} \equiv\left(\frac{\partial y^{\alpha}}{\partial x^{\beta}}\right) \gamma^{\beta}$ then satisfy the anticommutation relations

$\left\{\beta^{\mu}, \beta^{\nu}\right\}=2 g^{\mu \nu},\left\{\beta^{\mu}, \beta^{4}\right\}=0$,

with $g^{\mu \nu}=\frac{\partial y^{\mu}}{\partial x^{\alpha}} \frac{\partial y^{\nu}}{\partial x^{\beta}} \eta^{\alpha \beta}$. Suppose that $\psi(x)$ is a solution of (2.13); if one writes $\psi=\left(1 \pm i \beta^{4}\right) \chi$, then $\chi$ satisfies the following equation:

$$
\left(\beta^{\mu} \frac{\partial}{\partial y^{\mu}}-2 H \beta^{4}-m\right) \chi=0
$$

this is the Gürsey-Lee equation [42]. This equation turns to the usual Dirac equation in the de Sitter space by choosing a local vierbein $e_{\mu}^{\alpha}$ at every point of de Sitter space and by setting $\gamma^{\mu}(X)=V \beta^{\mu}(y) V^{-1}$, then under the $V$ transformation, one obtains

$\left(\gamma^{\mu}(X) \nabla_{\mu}-m\right) \Psi(X)=0$.

Note that $\beta^{4}=\gamma_{\alpha} x^{\alpha}$ is related to the constant matrix $\gamma^{4}$ by $\gamma^{4}=V \beta^{4} V^{-1}$.

Any linear combination of $\left(1+i \beta^{4}\right) \chi$ and $\left(1-i \beta^{4}\right) \chi$ also satisfies the Gürsey-Lee equation

$\psi(x)=\frac{a_{1}}{2}\left(1+i \beta^{4}\right) \chi+\frac{a_{2}}{2}\left(1-i \beta^{4}\right) \chi$,

where the coefficients $a_{1}$ and $a_{2}$ are fixed by the normalization condition.

Now the relation between the spinor fields defined in embedding space and intrinsic space can be written as

$\psi(x)=\frac{a_{1}}{2} V^{-1}\left(1+i \gamma^{4}\right) \Psi(X)+\frac{a_{2}}{2} V^{-1}\left(1-i \gamma^{4}\right) \Psi(X)$,

or equivalently,

$\Psi(X)=\left[\frac{1}{2 a_{1}}\left(1+i \gamma^{4}\right)+\frac{1}{2 a_{2}}\left(1-i \gamma^{4}\right)\right] V \psi(x)$,

Similarly, for spin- $\frac{3}{2}$ field one obtains

$$
\begin{aligned}
\Psi_{\mu}(X) & =\left[\frac{1}{2 a_{1}}\left(1+i \gamma^{4}\right)+\frac{1}{2 a_{2}}\left(1-i \gamma^{4}\right)\right] V \frac{\partial x^{\alpha}}{\partial X^{\mu}} \psi_{\alpha}(x), \\
& \equiv \mathcal{A} \frac{\partial x^{\alpha}}{\partial X^{\mu}} \psi_{\alpha}(x),
\end{aligned}
$$

where $\mathcal{A}$ matrix is given by

$\mathcal{A}=\left[\frac{1}{2 a_{1}}\left(1+i \gamma^{4}\right)+\frac{1}{2 a_{2}}\left(1-i \gamma^{4}\right)\right] V$,

In this case, the covariant derivatives in de Sitter intrinsic space are related to the derivative in embedding space as [43]

$$
\begin{gathered}
\nabla_{\mu} \Psi_{\nu}(X)=\mathcal{A} \frac{\partial x^{\alpha}}{\partial X^{\mu}} \frac{\partial x^{\beta}}{\partial X^{\nu}}\left(\partial_{\alpha}^{T} \psi_{\beta}-x_{\beta} \psi_{\alpha}\right) \\
\nabla_{\mu} \nabla_{\nu} \Psi_{\rho}(X)=\mathcal{A} \frac{\partial x^{\alpha}}{\partial X^{\mu}} \frac{\partial x^{\beta}}{\partial X^{\nu}} \frac{\partial x^{\gamma}}{\partial X^{\rho}} \operatorname{Trpr} \partial_{\alpha}^{T} \operatorname{Trpr} \partial_{\beta}^{T} \psi_{\gamma} \\
=\mathcal{A} \frac{\partial x^{\alpha}}{\partial X^{\mu}} \frac{\partial x^{\beta}}{\partial X^{\nu}} \frac{\partial x^{\gamma}}{\partial X^{\rho}}\left[\partial_{\alpha}^{T}\left(\partial_{\beta}^{T} \psi_{\gamma}-x_{\gamma} \psi_{\beta}\right)\right. \\
\left.-x_{\beta}\left(\partial_{\alpha}^{T} \psi_{\gamma}-x_{\gamma} \psi_{\alpha}\right)-x_{\gamma}\left(\partial_{\beta}^{T} \psi_{\alpha}-x_{\alpha} \psi_{\beta}\right)\right],
\end{gathered}
$$

Trpr is the abbreviation of the transverse projection. Therefore, the two-point function in the de Sitter space is related to the embedding space one by

$$
W_{\mu \mu^{\prime}}=\frac{\partial x^{\alpha}}{\partial X^{\mu}} \frac{\partial x^{\prime \alpha^{\prime}}}{\partial X^{\prime \mu^{\prime}}} \mathcal{A} \mathcal{S}_{\alpha \alpha^{\prime}} \mathcal{A}^{t}
$$


in this equation $\mathcal{S}_{\alpha \alpha^{\prime}}$ is defined by equation (5.15) and $\mathcal{A}^{t}$ is

$\mathcal{A}^{t}=\mathcal{A}^{\dagger} \gamma^{0} \gamma^{4}=\left[\frac{1}{2 a_{1}}\left(1+i \gamma^{4}\right)+\frac{1}{2 a_{2}}\left(1-i \gamma^{4}\right)\right] V^{t}$.

On the other hand, one can use three basic tensors to expand any maximally symmetric bitensor, these tensors are defined by [22]

$n_{\mu}=\nabla_{\mu} z\left(x, x^{\prime}\right), \quad n_{\mu^{\prime}}=\nabla_{\mu^{\prime}} z\left(x, x^{\prime}\right)$,

and the parallel propagator

$g_{\mu v^{\prime}}=-c^{-1}(\mathcal{P}) \nabla_{\mu} n_{v^{\prime}}+n_{\mu} n_{v^{\prime}}$.

The coefficients in this expansion are functions of the geodesic distance $z\left(x, x^{\prime}\right)$, between the points $x$ and $x^{\prime}$. It is de Sitter invariant and can be defined by an unique analytic extension. In this sense, these fundamental tensors form a complete set.

If $\mathcal{P} \equiv-x \cdot x^{\prime}$, then one can write

$\left\{\begin{array}{l}\mathcal{P}=\cosh z, \text { if } x \text { and } x^{\prime} \text { are timelike separated, } \\ \mathcal{P}=\cos z, \text { if } x \text { and } x^{\prime} \text { are spacelike separated. }\end{array}\right.$

In ambient space notations these tensors become

$\partial_{\alpha}^{T} z\left(x, x^{\prime}\right), \quad \partial_{\beta^{\prime}}^{\prime T} z\left(x, x^{\prime}\right), \quad \theta_{\alpha} \cdot \theta_{\beta^{\prime}}^{\prime}$.

For $\mathcal{P}=\cos z$, one can find

$$
\begin{aligned}
& n_{\mu}=\frac{\partial x^{\alpha}}{\partial X^{\mu}} \partial_{\alpha}^{T} z\left(x, x^{\prime}\right)=\frac{\partial x^{\alpha}}{\partial X^{\mu}} \frac{\left(x^{\prime} \cdot \theta_{\alpha}\right)}{\sqrt{1-\mathcal{P}^{2}}}, \\
& n_{v^{\prime}}=\frac{\partial x^{\prime \beta^{\prime}}}{\partial X^{\prime \nu^{\prime}}} \partial_{\beta^{\prime}}^{T} z\left(x, x^{\prime}\right)=\frac{\partial x^{\prime \beta^{\prime}}}{\partial X^{\prime \nu^{\prime}}} \frac{\left(x \cdot \theta_{\beta^{\prime}}^{\prime}\right)}{\sqrt{1-\mathcal{P}^{2}}}, \\
& \nabla_{\mu} n_{v^{\prime}}=\frac{\partial x^{\alpha}}{\partial X^{\mu}} \frac{\partial x^{\prime \beta^{\prime}}}{\partial X^{\prime \nu^{\prime}}} \theta_{\alpha}^{\varrho} \theta_{\beta^{\prime}}^{\prime \gamma^{\prime}} \partial_{\varrho}^{T} \partial_{\gamma^{\prime}}^{T} z\left(x, x^{\prime}\right) \\
& \quad=c(\mathcal{P})\left[n_{\mu} n_{v^{\prime}} \mathcal{P}-\frac{\partial x^{\alpha}}{\partial X^{\mu}} \frac{\partial x^{\prime \beta^{\prime}}}{\partial X^{\prime \nu^{\prime}}} \theta_{\alpha} \cdot \theta_{\beta^{\prime}}^{\prime}\right],
\end{aligned}
$$

where $c^{-1}(\mathcal{P})=-\frac{1}{\sqrt{1-\mathcal{P}^{2}}}$. In the case of $\mathcal{P}=\cosh z$, one has $c(\mathcal{P})=-\frac{i}{\sqrt{1-\mathcal{P}^{2}}}$, and hence in both cases, one obtains

$g_{\mu v^{\prime}}+(\mathcal{P}-1) n_{\mu} n_{v^{\prime}}=\frac{\partial x^{\alpha}}{\partial X^{\mu}} \frac{\partial x^{\prime \beta^{\prime}}}{\partial X^{\prime v^{\prime}}} \theta_{\alpha} \cdot \theta^{\prime}{ }_{\beta^{\prime}}$.

After making use of the above relations and (6.7), the twopoint function (5.15) in the de Sitter space is found as

$$
\begin{aligned}
W_{\mu \mu^{\prime}}= & \mathcal{A}\left\{g_{\mu \mu^{\prime}}+(\mathcal{P}-1) n_{\mu} n_{\mu^{\prime}}\right. \\
+ & \left(\frac{1}{10}\left(-\nabla_{\mu}-\gamma_{\mu}^{T}(X) \not x\right)(1-2 \not x)+\frac{1}{4} \gamma_{\mu}^{T}(X)\right) \\
& \times\left[-3 \not x\left(X \cdot g_{\mu^{\prime}}^{\prime}\right)+\left(g_{\mu^{\prime}}^{\prime} \cdot \gamma_{\mu}^{T}(X)\right)\right. \\
& \left.\left.-\frac{1}{5} X_{\mu^{\prime}}(7 \not x-14)-\gamma_{\mu^{\prime}}^{T}(X)\right]\right\} \mathcal{S}_{1} \mathcal{A}^{t},
\end{aligned}
$$

where $\mathcal{S}_{1}$ is given by (5.10). This two-point function is obviously dS-invariant.

\section{Conclusions}

Conformal theories and conformal techniques have been used for a long time in physics (see for example [44] and references therein), e.g., in the gravitational physics, the main motivation of considering such theories is to achieve a proper theory of quantum gravity. As one knows, the quantum theory of gravity based on Einstein equation is not renormalizable [45], however, it is proved that the conformal theories of gravity are better to renormalize [46]. On the other hand, the gravitational field is long range and propagates with the speed of light, thus in the linear approximation, it is expected that the equations governing its dynamic must be conformally invariant, whereas, the Einstein equation is not conformally invariant equation. Moreover, within the standard theory of gravity one needs to introduce a huge amount of dark and non-detectable matter and energy to explain some extraordinary phenomena [47], while the conformally invariant Weyl gravity can address some of these large scale issues.

On the other hand, as is well known, the powerful AdS/CFT conjecture makes a relation between the quantum aspects of gravity in anti-de Sitter space and dual conformal theory which lives on its boundary. But as mentioned our universe governed by a non-vanishing positive cosmological constant and hence, a proper dictionary of $\mathrm{dS} / \mathrm{CFT}$ is desired. It is believed that a better understanding of higher-spin and gauge theories in the de Sitter space will help one to find a proper dS/CFT correspondence [48].

The massless spin- $\frac{3}{2}$ (gravitino) field is supposed to be the fermionic partner of gravitational field, which is used in supergravity to unify gravitational (spin-2) and nongravitational (spin-1) forces [49]. In this paper by choosing the de Sitter space as the background, and with the aid of the background symmetries which well appear in dS group, we considered the massless spin- $\frac{3}{2}$ field by constructing its conformal invariant field equation. The solutions and two-point functions are also found. 
Acknowledgments We would like to thank the referees for their comments and criticisms that resulted in improvement of the manuscript. We are grateful to M.R. Enaiati, M. Parsamehr for useful discussions. MRT would also like to acknowledge M. Tanhayi for comments on higher-spin fields. MRT financially supported by Islamic Azad University Central Tehran Branch.

Open Access This article is distributed under the terms of the Creative Commons Attribution License which permits any use, distribution, and reproduction in any medium, provided the original author(s) and the source are credited.

Funded by $\mathrm{SCOAP}^{3}$ / License Version CC BY 4.0.

\section{Appendix A: A note on UIR of de Sitter group}

The unitary irreducible representation of the dS group are classified according to a pair $(p, q)$ of the possible spectral values of the Casimir operators as follows [30,31]:

$$
\begin{aligned}
\left\langle Q_{p}^{(1)}\right\rangle= & (-p(p+1)-(q+1)(q-2)) \mathrm{I} 1, \\
& \left\langle Q_{p}^{(2)}\right\rangle=(-p(p+1) q(q-1)) \mathrm{I} 1 .
\end{aligned}
$$

For each kind of field, the possible range of the parameters $q$ and $p$ (where $2 p \in \mathbb{N}$ and $q \in \mathbb{C}$ ) indicates the unitary and also irreducible representation, generally they characterize three types of UIR in $\mathrm{dS}$, which can be listed as follows:

1. Principal series $U_{p, q}$

$$
\left\{\begin{array}{l}
p=s, \text { and } q=\frac{1}{2}+i v, \quad \text { for } v \geq 0, \quad s=0,1,2, \ldots \\
p=s, \text { and } q=\frac{1}{2}+i v, \quad \text { for } v>0, \quad s=1 / 2,3 / 2 \ldots
\end{array}\right.
$$

which also called the massive representation because in the flat limit they tend to the massive representation of the Poincaré group with spin $s$.

2. Complementary series $V_{p, q}$

$$
\left\{\begin{array}{l}
p=s, \quad \text { and } q=\frac{1}{2}+v, \quad \text { for } 0<|v|<3 / 2, \quad s=0, \\
p=s, \quad \text { and } q=\frac{1}{2}+v, \quad \text { for } 0<|v|<1 / 2, \quad s=1,2, \ldots
\end{array}\right.
$$

in this case $v \in \mathbb{R}$, and the massless conformally coupled scalar field in this series has the Poincaré limit.

3. Discrete series $\Pi_{p, q}^{ \pm}$: in this case the only representation which has the Poincaré limit is $p=q=s$. This series represents the massless fields.
In $\mathrm{dS}$ space the massless field with spin- $\frac{1}{2}$ is associated with a discrete series $\prod_{p, s}^{ \pm}$and their UIRs are $\prod_{\frac{1}{2}, \frac{1}{2}}^{ \pm}$, where $p=q=s=\frac{1}{2}$ correspond to $\left\langle Q_{\frac{1}{2}}^{(1)}\right\rangle=\frac{3}{2}$ and these two representations have a Minkowskian interpretation. For spin- $\frac{3}{2}$ field, the two UIRs $\Pi_{\frac{3}{2}, \frac{3}{2}}^{ \pm}$, have a Minkowskian interpretation. For example $\Pi_{\frac{3}{2}, \frac{1}{2}}^{ \pm}$have no corresponding flat limit. It is proved that for every massless representation of the Poincaré group there exists only one corresponding representation in the conformal group [50,51]. In the massless case, conformal invariance leads one to deal with the discrete series representations and their lower limits of the universal covering of the conformal group. The conformal group is locally isomorphic to $S O(2,4)$ and its compact subgroup is $S O(2) \times S O(4)$, where the generator of $S O(2)$ is the conformal energy. The unitary irreducible representations of the conformal group are denoted in the sequel by $\mathcal{C}\left( \pm E_{0}, j_{1}, j_{2}\right)$, where $\left(j_{1}, j_{2}\right) \in \mathbb{N} / 2 \times \mathbb{N} / 2$ labels the UIRs of $S U(2) \times S U(2)$ and $E_{0}$ stands for the positive (resp. negative) conformal energy. The direct sum of two UIRs $C(j+1, j, 0)$ and $C(-j-1, j, 0)$ of the conformal group with positive and negative energy and $j=\frac{1}{2}$ for spinor field $j=\frac{3}{2}$ for the spin- $\frac{3}{2}$ field is a unique extension of the representation $\prod_{j, j}^{+}$. We shall denote the massless Poincaré UIRs by $P^{>}(0, j)$ and $P^{<}(0, j)$ which, respectively, are the positive and negative energies representation with positive helicity. The following diagrams illustrate these relations:

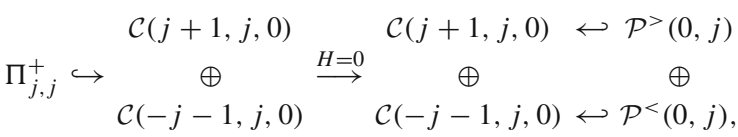

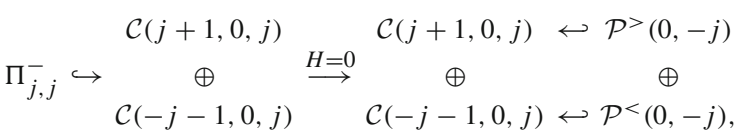

where the arrows $\hookrightarrow$ designate unique extension.

\section{Appendix B: Some useful relations}

In this appendix, some useful relations are given which are used in this paper: 


$$
\begin{array}{ll}
{\left[\partial_{\alpha}^{T}, \partial_{\beta}^{T}\right]=x_{\beta} \partial_{\alpha}^{T}-x_{\alpha} \partial_{\beta}^{T},} & {\left[\partial_{\alpha}^{T}, x_{\beta}\right]=\theta_{\alpha \beta},} \\
{\left[\gamma_{\alpha}^{T}, x_{\beta}\right]=0,} & {\left[\gamma_{\alpha}^{T}, \partial_{\beta}^{T}\right]=-\left(\theta_{\alpha \beta} \not x+x_{\alpha} \gamma_{\beta}^{T}\right),} \\
Q_{0}=-\left(\partial_{\alpha}^{T}\right)^{2}, & Q_{0} \partial_{\alpha}^{T}=\partial_{\alpha}^{T} Q_{0}+2 \partial_{\alpha}^{T}+2 x_{\alpha} Q_{0}, \\
Q_{0} x_{\alpha}=x_{\alpha} Q_{0}-4 x_{\alpha}-2 \partial_{\alpha}^{T}, & \gamma_{\alpha}^{T}=\Theta_{\alpha}^{\beta} \gamma_{\beta}=\gamma_{\alpha}+x_{\alpha} x \cdot \gamma, \\
Q_{\frac{3}{2}}^{(1)} \mathcal{D}_{\alpha}^{\left(\frac{3}{2}\right)}=\mathcal{D}_{\alpha}^{\left(\frac{3}{2}\right)} Q_{\frac{1}{2}}^{(1)}, & \gamma_{\alpha}^{T} \not x=-\not x \gamma_{\alpha}^{T}, \\
Q_{\frac{3}{2}}^{(1)} \partial_{\alpha}^{T}=\partial_{\alpha}^{T}\left(Q_{0}+\not x \partial^{T}\right), & \partial^{T} \mathcal{D}_{\alpha}^{\left(\frac{3}{2}\right)}=\mathcal{D}_{\alpha}^{\left(\frac{3}{2}\right)} \not \partial^{T}-\not x \partial_{\alpha}^{T}-x_{\alpha} \partial^{T}-4 x_{\alpha} \not x+3 \gamma_{\alpha}^{T}, \\
\not x \partial^{T} x_{\alpha}=x_{\alpha} \not x \partial^{T}+\not x \gamma_{\alpha}^{T}, & Q_{0} \not x \partial^{T}=\not x \partial^{T} Q_{0}, \\
Z_{\alpha}^{T}=Z_{\alpha}+x_{\alpha} x \cdot Z, & Q_{0} Z_{\alpha}^{T}=Z_{\alpha}^{T} Q_{0}-2 x_{\alpha} Z \cdot \partial^{T}-4 x_{\alpha} x \cdot Z, \\
\partial^{T} \cdot Z^{T}=Z \cdot \partial^{T}+4 x \cdot Z, & Z^{T}=\not Z+\not x x \cdot Z, \\
\partial^{T}\left(Z \cdot \partial^{T}\right)=-2 \not x\left(Z \cdot \partial^{T}\right)+\not x(x \cdot Z), & \partial^{T}(x \cdot Z)=-\not x(x \cdot Z)+\not Z^{T}, \\
\left(x \cdot \theta_{\alpha^{\prime}}\right)\left(x \cdot \theta^{\prime \alpha^{\prime}}\right)=\left(x \cdot x^{\prime}\right)^{2}-1, & \partial_{\alpha}^{T}\left(x \cdot \theta_{\beta^{\prime}}^{\prime}\right)=\theta_{\alpha} \cdot \theta_{\beta^{\prime}}^{\prime}, \\
\partial_{\alpha}^{T}\left(x^{\prime} \cdot \theta_{\beta}\right)=x_{\beta}\left(x^{\prime} \cdot \theta_{\alpha}\right)-Z \theta_{\alpha \beta}, & \partial_{\alpha}^{T}\left(\theta_{\beta} \cdot \theta_{\beta^{\prime}}^{\prime}\right)=x \beta\left(\theta_{\alpha} \cdot \theta_{\beta^{\prime}}^{\prime}\right)+\theta_{\alpha \beta}\left(x \cdot \theta_{\beta^{\prime}}^{\prime}\right), \\
x_{\alpha}\left(Z . \partial^{T}\right)=\left(Z . \partial^{T}\right) x_{\alpha}-Z_{\alpha}^{T}, &
\end{array}
$$

and also the following identities are used:

$$
\begin{aligned}
Q_{0} \not x\left(Z \cdot \partial^{T}\right)= & \not x\left(Z \cdot \partial^{T}\right) Q_{0}-2 \not x\left(Z \cdot \partial^{T}\right) \\
& +2 \not x(x \cdot Z) Q_{0}-2 \not \partial^{T}\left(Z \cdot \partial^{T}\right), \\
\partial^{T} Z^{T}= & \not Z^{T} \not \partial^{T}+\not x \not Z^{T}+4 x \cdot Z, Q_{0} \not x(x \cdot Z) \\
= & \not x x \cdot Z Q_{0}-8 \not x x \cdot Z-2 \not x\left(Z \cdot \partial^{T}\right) \\
& -2 \not \partial^{T}(x \cdot Z), \\
Q_{0} \mathcal{D}_{\alpha}^{\left(\frac{3}{2}\right)}= & \mathcal{D}_{\alpha}^{\left(\frac{3}{2}\right)} Q_{0}-4 \partial_{\alpha}^{T}+4 \gamma_{\alpha}^{T} \not x-2 \gamma_{\alpha}^{T} \not \partial^{T} \\
& -2 x_{\alpha} \not x \not \partial^{T} .
\end{aligned}
$$

\section{Appendix C: Conformally invariant wave equation}

In this appendix we give some details of CI equations. As was shown, we obtained the following equation from (3.2) by choosing $n=1$ :

$\left(Q_{0}-2\right) \Phi_{\alpha}=0$,

note $\Phi_{\alpha}$ is defined on conformal space. Multiply (C.1) by $x_{\alpha}$ from the left and after making use of the relations given in Appendix B, leads one to write

$Q_{0} x \cdot \Phi+2 x \cdot \Phi+2 \partial^{T} \cdot \Phi=0$.

The transversality condition on the cone yields $u_{a} \Phi^{a b \cdots}=0=u_{5} \Phi^{5}+u_{\alpha} \Phi^{\alpha}$,

$x_{5}\left(\Phi^{5}+x . \Phi\right)=0$,

then by acting $\left(Q_{0}-2\right)$ on this relation, we obtain

$\left(Q_{0}-2\right) x \cdot \Phi=0$.

By using (C.3) in (C.2) one has

$\partial^{T} \cdot \Phi=-2 x \cdot \Phi$.
The divergence of $\Psi_{\alpha}$ defined in (3.8) reads

$\partial^{T} \cdot \Psi=x_{5}\left[\partial^{T} . \Phi+4 x . \Phi\right]=2 x_{5} x \cdot \Phi$,

by using (C.3) we can write

$\left(Q_{0}-2\right) \partial^{T} \cdot \Psi=0$.

Now, if we act $\left(Q_{0}-2\right)$ on the $\Psi_{\alpha}$ we have

$$
\begin{aligned}
& \left.\left(Q_{0}-2\right) \Psi_{\alpha}=x_{5}\left[\left(Q_{0}-2\right) \Phi_{\alpha}+\left(Q_{0}-2\right) x_{\alpha} x . \Phi\right)\right] \\
& =x_{5}\left[x_{\alpha} Q_{0} x \cdot \Phi-4 x_{\alpha} x . \Phi-2 \partial_{\alpha}^{T}(x . \Phi)-2 x_{\alpha} x . \Phi\right] \\
& =x_{5}\left[-4 x_{\alpha} x \cdot \Phi-2 \partial^{T}{ }_{\alpha}(x \cdot \Phi)\right],
\end{aligned}
$$

which has been obtained from (3.8). Finally the CI spin- $\frac{3}{2}$ equation can be written as

$\left(Q_{0}-2\right) \Psi_{\alpha}+2 x_{\alpha} \partial^{T} \cdot \Psi+\partial_{\alpha}^{T} \partial^{T} \cdot \Psi=0$.

\section{Appendix D: Generalized Legendre functions}

The generalized Legendre $P_{\lambda}^{d+1}$ introduced in equations (5.10) is defined by [52]

$$
\begin{aligned}
& P_{\lambda}^{d+1}(Z)=\frac{\Gamma(d / 2)}{\sqrt{\pi} \Gamma\left(\frac{d-1}{2}\right)} \\
& \quad \times \int_{0}^{\pi}\left[Z+\left(Z^{2}-1\right)^{1 / 2} \cos t\right]^{\lambda}(\sin t)^{(d-2)} \mathrm{d} t .
\end{aligned}
$$

This function is proportional to the Gegenbauer function $C_{\lambda}^{k}(Z)$ of the first kind, thus

$$
\begin{aligned}
P_{\lambda}^{d+1}(Z) & =\frac{\Gamma(d-1) \Gamma(\lambda+1)}{\Gamma(\lambda+d-1)} C_{\lambda}^{(d-1) / 2}(Z) \\
& =F\left(\lambda+d-1,-\lambda ; \frac{d}{2} ; \frac{1-Z}{2}\right),
\end{aligned}
$$


where

$$
\begin{aligned}
C_{\lambda}^{k}(Z)= & \frac{\Gamma(\lambda+2 k)}{\Gamma(\lambda+1) \Gamma(2 k)} F \\
& \times\left(\lambda+2 k,-\lambda, ; k+\frac{1}{2} ; \frac{1-Z}{2}\right),
\end{aligned}
$$

and $F$ is the hypergeometric function. The $P_{\lambda}^{(d+1)}(Z)$ and Legendre functions $P_{v}^{\mu}(Z)$ are related by

$$
P_{\lambda}^{d+1}(Z)=2^{(d-2) / 2} \Gamma\left(\frac{d}{2}\right)\left(Z^{2}-1\right)^{(2-d) / 4} P_{\lambda+(d-2) / 2}^{(2-d) / 2}(Z) .
$$

\section{Appendix E: Wave equation of spin-3/2 field}

Studying higher-spin fields has a rich history dating back to the early work of Fierz-Pauli (which based on the positivity of energy after first quantization) and Bargmann-Wigner (which the positivity could be replaced by the requirement of being an UIR) and many others (see for example [53-55] and references therein). One approach of studying free higherspin fields is the analysis of the corresponding relativistic wave equations which could be based on the BargmannWigner scheme. ${ }^{3}$ By this method a manifestly covariant differential equation is associated with a given UIR of the Poincaré group and the positive energy solutions of the equations transform according to the corresponding UIR [9]. For example, one can derive Maxwell's equations via two equations of massless spin-1/2. The solutions of the BargmannWigner equations are actually the fields that transform under the symmetric group as totally symmetric multi-spinors of definite mass and spin. In anti-de Sitter space, covariant massless fields for half-integer spin have been considered [11-13]. In this appendix, we extend this approach of finding covariant massless field in de Sitter space, by explicitly writing the massless spin-3/2 field equation in terms of vector and spin$1 / 2$ field equations. The mathematical details go as follows.

The action of $S_{\alpha \beta}$ on a tensor field of $\operatorname{rank} l, \Psi_{\gamma_{1} \ldots \gamma_{l}}(x)$, is given by

$$
\begin{aligned}
S_{\alpha \beta}^{(l)} \Psi_{\gamma_{1} \ldots \gamma_{l}}= & -i \sum_{i=1}^{l}\left(\eta_{\alpha \gamma_{i}} \Psi_{\gamma_{1} \ldots\left(\gamma_{i} \rightarrow \beta\right) \ldots \gamma_{l}}\right. \\
& \left.-\eta_{\beta \gamma_{i}} \Psi_{\gamma_{1} \ldots\left(\gamma_{i} \rightarrow \alpha\right) \ldots \gamma_{l}}\right) .
\end{aligned}
$$

On the other hand, for a half-integer spin field with spin $s=l+\frac{1}{2}$ which is represented by a four-component spinortensor $\Psi_{\gamma_{1} \ldots \gamma_{l}}^{i}$ with spinor index $i=1,2,3$, 4, one has

$S_{\alpha \beta}^{(s)}=S_{\alpha \beta}^{(l)}+S_{\alpha \beta}^{\left(\frac{1}{2}\right)}, \quad$ with $\quad S_{\alpha \beta}^{\left(\frac{1}{2}\right)}=-\frac{i}{4}\left[\gamma_{\alpha}, \gamma_{\beta}\right]$,

\footnotetext{
${ }^{3}$ We thank a very conscientious referee who suggested this method.
}

where the Dirac gamma matrices are satisfied by (2.6), and they are explicitly given by

$\gamma^{0}=\left(\begin{array}{cc}I & 0 \\ 0 & -I\end{array}\right), \gamma^{4}=\left(\begin{array}{cc}0 & I \\ -I & 0\end{array}\right)$,

$\gamma^{1}=\left(\begin{array}{cl}0 & i \sigma^{1} \\ i \sigma^{1} & 0\end{array}\right), \gamma^{2}=\left(\begin{array}{cl}0 & -i \sigma^{2} \\ -i \sigma^{2} & 0\end{array}\right)$,

$\gamma^{3}=\left(\begin{array}{cc}0 & i \sigma^{3} \\ i \sigma^{3} & 0\end{array}\right)$,

where $\sigma_{i}$ are Pauli matrices and $I$ is a $2 \times 2$ unit matrix. After doing some calculations one can show that for a $l$-rank tensor field $\Psi_{\gamma_{1} \ldots \gamma_{l}}(x)$, the following relations hold:

$$
\begin{aligned}
Q_{l}^{(1)} \Psi= & Q_{0}^{(1)} \Psi-2 \Sigma_{1} \partial x . \Psi+2 \Sigma_{1} x \partial . \Psi \\
& +2 \Sigma_{2} \eta \Psi^{\prime}-l(l+1) \Psi,
\end{aligned}
$$

where

$$
\begin{aligned}
& Q_{l}^{(1)}=-\frac{1}{2} L_{\alpha \beta}^{(l)} L^{\alpha \beta(l)} \\
& \quad=-\frac{1}{2} M_{\alpha \beta} M^{\alpha \beta}-\frac{1}{2} S_{\alpha \beta}^{(l)} S^{\alpha \beta(l)}-M_{\alpha \beta} S^{\alpha \beta(l)}, \\
& M_{\alpha \beta} S^{\alpha \beta(l)} \Psi(x)=2 \Sigma_{1} \partial x . \Psi-2 \Sigma_{1} x \partial . \Psi-2 l \Psi, \\
& \frac{1}{2} S_{\alpha \beta}^{(l)} S^{\alpha \beta(l)} \Psi=l(l+3) \Psi-2 \Sigma_{2} \eta \Psi^{\prime},
\end{aligned}
$$

where $Q_{0}^{(1)}=-\frac{1}{2} M_{\alpha \beta} M^{\alpha \beta}$ and $\Psi^{\prime}$ is the trace of $\Psi(x)$ and $\Sigma_{p}$ is the non-normalized symmetrization operator given by

$$
\begin{aligned}
& \Psi_{\alpha_{1} \ldots \alpha_{l-2}}^{\prime}=\eta^{\alpha_{l-1} \alpha_{l}} \Psi_{\alpha_{1} \ldots \alpha_{l-2} \alpha_{l-1} \alpha_{l}}, \\
& \left(\Sigma_{p} A B\right)_{\alpha_{1} \ldots \alpha_{l}}=\sum_{i_{1}<i_{2}<\ldots<i_{p}} A_{\alpha_{i_{1}} \alpha_{i_{2}} \ldots \alpha_{i_{p}}} B_{\alpha_{1} \ldots \alpha_{i_{1}} \ldots \alpha_{i_{2}} \ldots \alpha_{i_{p}} \ldots \alpha_{l}} .
\end{aligned}
$$

In the case of a half-integer spin field, $s=l+\frac{1}{2}$, the $S_{\alpha \beta}^{\left(\frac{1}{2}\right)}$ acts only upon the index $i$

$\mathcal{S}_{\alpha \beta}^{\left(\frac{1}{2}\right)} \mathcal{S}^{\alpha \beta(l)} \Psi(x)=l \Psi(x)-\Sigma_{1} \gamma(\gamma \cdot \Psi(x))$,

and the Casimir operator becomes

$$
\begin{aligned}
Q_{s}^{(1)} & =-\frac{1}{2}\left(M_{\alpha \beta}+S_{\alpha \beta}^{(l)}+S_{\alpha \beta}^{\left(\frac{1}{2}\right)}\right)\left(M^{\alpha \beta}+S^{\alpha \beta(l)}+S^{\alpha \beta\left(\frac{1}{2}\right)}\right) \\
& =Q_{l}^{(1)}-\frac{5}{2}+\frac{i}{2} \gamma_{\alpha} \gamma_{\beta} M^{\alpha \beta}-S_{\alpha \beta}^{\left(\frac{1}{2}\right)} S^{\alpha \beta(l)} .
\end{aligned}
$$

Therefore one obtains

$$
\begin{aligned}
Q_{s}^{(1)} \Psi(x) & =\left(Q_{l}^{(1)}-l-\frac{5}{2}+\frac{i}{2} \gamma_{\alpha} \gamma_{\beta} M^{\alpha \beta}\right) \Psi(x)+\Sigma_{1} \gamma(\gamma \cdot \Psi(x)) \\
& =\left(-\frac{1}{2} M_{\alpha \beta} M^{\alpha \beta}+\frac{i}{2} \gamma_{\alpha} \gamma_{\beta} M^{\alpha \beta}-l(l+2)-\frac{5}{2}\right) \Psi(x)
\end{aligned}
$$




$$
\begin{aligned}
& -2 \Sigma_{1} \partial x \cdot \Psi(x)+2 \Sigma_{1} x \partial \cdot \Psi(x)+2 \Sigma_{2} \eta \Psi^{\prime}(x) \\
& +\Sigma_{1} \gamma(\gamma \cdot \Psi(x)) .
\end{aligned}
$$

Therefore, any higher-spinor field, $s=l+\frac{1}{2}$, can indeed be decomposed into a tensor and spinor field. In our case in this paper it was shown that $s=\frac{3}{2}$ field can be written in terms of a spinor field and a polarization vector with total four degrees of freedom in the de Sitter space.

\section{References}

1. A.G. Riess, et al., Observational evidence from supernovae for an accelerating universe and a cosmological constant. Astron. J. 116, 1009 (1998). arxiv:astro-ph/9805201

2. A. Linde, Particle physics and inflationary cosmology. Harwood Academic Publishers, The Netherlands (1990)

3. T.S. Bunch, P.C.W. Davies, Quantum field theory in de Sitter space: renormalization by point-splitting. Proc. R. Soc. Lond. A 360, 117 (1978)

4. B. Allen, Vacuum states in de Sitter space. Phys. Rev. D 32, 3136 (1985)

5. J. Bros, U. Moschella, Two-point functions and quantum field in the de Sitter universe. Rev. Math. Phys. 8, 327 (1996). arxiv:gr-qc/9511019

6. N. A. Chernikov, E. A. Tagirov, Quantum theory of scalar field in de Sitter space-time. Ann. Inst. Henri Poincaré IX, 109 (1968)

7. M.V. Takook, Entropy of quantum fields in de Sitter space-time. arXiv: 1306.3575 [gr-qc]

8. M.V. Takook, Quantum field theory in de Sitter universe: ambient space formalism. arXiv:1403.1204v2 [grqc]

9. V. Bargmann, E.P. Wigner, Group theoretical discussion of relativistic wave equations. Proc. Natl. Acad. Sci. 34, 211 (1948)

10. D. Luriè, Particles and fields. Interscience Publishers, New York (1968)

11. J. Fang, C. Fronsdal, Massless, half-integral spin fields in de Sitter space. Phys. Rev. D 22, 1361 (1980)

12. M. Lesimple, Homogeneous propagators for massless half-integer spin fields in 3+2 de Sitter space. Lett. Math. Phys. 18 (1989)

13. M. Lesimple, Construction of homogeneous propagators for massive half-integer spin fields in $3+2$ de Sitter space, with implications for the massless limit. 15, 143 (1988)

14. M.V. Takook, A. Azizi, E. Babaian, Covariant quantization of "massive" spin-3/2 fields in the de Sitter space. Eur. Phys. J. C 72, 2026 (2012). arXiv:1206.1997

15. A. Azizi, M. Amiri, "Massless" spin- $3 / 2$ fields in the de Sitter space. Eur. Phys. J. C 74, 2768 (2014). arXiv:1401.6381v1

16. R.M. Wald, General relativity (The University of Chicago Press, Chicago, 1984)

17. W. Siegel, All free conformal representations in all dimensions. Int. J. Mod. Phys. A 4, 2015 (1989)

18. R.R. Metsaev, Fermionic fields in the d-dimensional anti-de Sitter spacetime. Phys. Lett. B 419, 49 (1998)

19. R.R. Metsaev, Free totally (anti)symmetric massless fermionic fields in d-dimensional anti-de Sitter space. Class. Quant. Grav. 14, 115 (1997)

20. J.P. Gazeau, M.V. Takook, Massive vector field in the de Sitter space. J. Math. Phys. 41, 5920 (2000). arxiv:gr-qc/9912080

21. C. Gabriel, P. Spindel, Massive spin-2 propagator on de Sitter space. J. Math. Phys. 38, 622 (1997). arxiv:hep-th/9912054

22. T. Garidi, J.P. Gazeau, M.V. Takook, Massive spin-2 field in de Sitter space. J. Math. Phys. 44, 3838 (2003). arxiv:hep-th/0302022
23. M. Laoues, Massless particles in arbitrary dimensions. Rev. Math. Phys. 10, 1079 (1998). arxiv:hep-th/9806101

24. T. Garidi, J.P. Gazeau, S. Rouhani, M.V. Takook, Massless vector field in de Sitter universe. J. Math. Phys. 49, 032501 (2008). arxiv:gr-qc/0608004

25. J. Bros, J.P. Gazeau, U. Moschella, Quantum field theory in the de sitter universe. Phys. Rev. Lett. 73, 1746 (1994)

26. J.P. Gazeau, J. Renaud, M.V. Takook, Gupta-Bleuler quantization for minimally coupled scalar field in de Sitter space. Class. Quant. Grav. 17, 1415 (2000). arxiv:gr-qc/9904023

27. S. Faci, E. Huguet, J. Queva, J. Renaud, Conformally covariant quantization of the Maxwell field in de Sitter space. Phys. Rev. D 80, 124005 (2009). arXiv:0910.1279 [gr-qc]

28. S. Faci, E. Huguet, J. Renaud, Conformal use of retarded Green's functions for the Maxwell field in de Sitter space. Phys. Rev. D 84, 124050 (2011). arXiv:1110.1177 [gr-qc]

29. P. Moylan, Unitary representations of the (4+1)-de Sitter group on irreducible representation spaces of the Poincaré group. J. Math. Phys. 24, 2706 (1983)

30. J. Dixmier, Bull. Soc. Math. France 89, 9 (1961)

31. B. Takahashi, Bull Soc. Math. France 91, 289 (1963)

32. P. Bartesaghi, J.P. Gazeau, U. Moschella, M.V. Takook, Dirac fields and thermal effects in the de Sitter universe. Class. Quant. Grav. 17, 4373 (2001)

33. M.V. Takook, "Thèorie quantique des champs pour des systèmes èl "ementaires "massifs" et de "masse nulle" sur l'espace-temps de de Sitter". Thèse de l'université Paris VI (1997)

34. E. Cunningham, The principle of relativity in electrodynamics and an extension thereof. Proc. Math. Soc. Lond. 8, 77 (1909) (H. Bateman, ibid 8, 223, 1910)

35. P.A.M. Dirac, Wave equations in conformal space. Ann. Math. 37, 429 (1936)

36. X. Bekaert, M. Grigoriev, Manifestly conformal descriptions and higher symmetries of bosonic singletons. SIGMA 6, 038 (2010). arXiv:0907.3195 [hep-th]

37. P. Arvidsson, R. Marnelius, Conformal theories including conformal gravity as gauge theories on the hypercone. arxiv:hep-th/0612060v1

38. S. Behroozi, S. Rouhani, M.V. Takook, M. R. Tanhayi, Conformally invariant wave-equations and massless fields in de Sitter space time. Phys. Rev. D 74, 124014 (2006). arxiv:gr-qc/0512105

39. M. Dehghani, S. Rouhani, M.V. Takook, M. R. Tanhayi, Conformally invariant massless spin-2 field in the de Sitter universe. Phys. Rev. D 77, 064028 (2008). arXiv:0805.2227

40. J.P. Gazeau, M. Hans, Conformally invariant wave equations in 3+2-de Sitter space. Suppl. Rend. Circ. Mat. Palemo Ser. II 21(21), 179 (1989)

41. C. Fronsdal, Singletons and massless integral-spin fields on de Sitter space. Phys. Rev. D 20, 848 (1979)

42. F. Gursey, T.D. Lee, Spin-1/2 wave equation in de Sitter space. Proc. Natl. Acad. Sci. USA 49, 179 (1963)

43. M.V. Takook, M.R. Tanhayi, Linear Weyl gravity in de Sitter universe. JHEP 12, 044 (2010). arXiv:0903.2670

44. Y. Nakayama, A lecture note on scale invariance vs conformal invariance. arXiv: 1302.0884

45. N.D. Birrel, P.C.W. Davies, Quantum fields in curved space (Cambridge University Press, Cambridge, 1982)

46. K.S. Stelle, Renormalization of higher-derivative quantum gravity. Phys. Rev. D 16, 953 (1977)

47. G. Bertonea, D. Hooperb, J. Silk, Particle dark matter: evidence, candidates and constraints. Phys. Rep. 405, 279 (2005). arxiv:hep-ph/0404175

48. D. Anninos, T. Hartman, A. Strominger, Higher spin realization of the dS/CFT correspondence. arxiv:1108.5735 [hep-th]

49. P. Van Nieuwenhuizen, Supergravity. Phys. Rep. 68, 189 (1981) 
50. A.O. Barut, A. Bohm, Reduction of a class of $\mathrm{O}(4,2)$ representation with respect to $\operatorname{So}(4,1)$ and $\operatorname{So}(3,2)$. J. Math. Phys. 11, 2938 (1970)

51. E. Angelopoulos, M. Laoues, Massless in $\mathrm{n}$ dimensions. Rev. Math. Phys. 10, 271 (1998). arxiv:hep-th/9806100

52. H. Bateman et al., Higher transcendental functions, vol. I (McGraw-Hill, New York, 1954)
53. M. A. Vasiliev, Higher-spin theory and space-time metamorphoses. arxiv: 1404.1948 [hep-th]

54. M.A. Vasiliev, Consistent equation for interacting gauge fields of all spins in (3+1)-dimensions. Phys. Lett. B 243, 378 (1990)

55. M.A. Vasiliev, Nonlinear equations for symmetric massless higher spin fields in (A)dS(d). Phys. Lett. B 567, 139 (2003). arxiv:hep-th/0304049 